\title{
Tissue structure modification in knee osteoarthritis by use of joint distraction: an open 1-year pilot study
}

\author{
Femke Intema, ${ }^{1}$ Peter M Van Roermund, ${ }^{2}$ Anne C A Marijnissen, ${ }^{1}$ Sebastian Cotofana, ${ }^{3}$ \\ Felix Eckstein, ${ }^{3}$ Rene M Castelein, ${ }^{2}$ Johannes W J Bijlsma, ${ }^{1}$ Simon C Mastbergen, ${ }^{1}$ \\ Floris P J G Lafeber ${ }^{1}$
}

- Additional data (supplementary figure) are published online only. To view these files please visit the journal online at (http://ard.bmj. com).

${ }^{1}$ Rheumatology and Clinical Immunology, University Medical Center Utrecht, Utrecht, The Netherlands 2Orthopaedics, University Medical Center Utrecht, Utrecht, The Netherlands ${ }^{3}$ Institute of Anatomy and Musculoskeletal Research, Paracelsus Medical University, Salzburg, Austria

\section{Correspondence to} Prof Floris Lafeber, Rheumatology \& Clinical Immunology, UMC Utrecht, F02.127, PO BOX 85500, 3508 GA Utrecht, The Netherlands; f.lafeber@umcutrecht.nl

Accepted 21 March 2011 Published Online First 12 May 2011

\section{(6) UNLOCKED}

This paper is freely available online under the BMJ Journals unlocked scheme, see http:// ard.bmj.com/info/unlocked.dtl

\begin{abstract}
Background Modification of joint tissue damage is challenging in late-stage osteoarthritis (OA). Few options are available for treating end-stage knee $\mathrm{OA}$ other than joint replacement.

Objectives To examine whether joint distraction can effectively modify knee joint tissue damage and has the potential to delay prosthesis surgery.

Methods 20 patients ( $<60$ years) with tibiofemoral $0 A$ were treated surgically using joint distraction. Distraction ( $\sim 5 \mathrm{~mm}$ ) was applied for 2 months using an external fixation frame. Tissue structure modification at 1 year of follow-up was evaluated radiographically (joint space width (JSW)), by MRI (segmentation of cartilage morphology) and by biochemical markers of collagen type II turnover, with operators blinded to time points. Clinical improvement was evaluated by Western Ontario and McMaster Universities Osteoarthritis Index (WOMAC) and Visual Analogue Scale (VAS) pain score.
\end{abstract}

Results Radiography demonstrated an increase in mean and minimum JSW (2.7 to $3.6 \mathrm{~mm}$ and 1.0 to $1.9 \mathrm{~mm} ; \mathrm{p}<0.05$ and $<0.01)$. MRI revealed an increase in cartilage thickness (2.4 to $3.0 \mathrm{~mm} ; \mathrm{p}<0.001)$ and a decrease of denuded bone areas (22\% to $5 \%$; $p<0.001)$. Collagen type II levels showed a trend towards increased synthesis $(+103 \% ; p<0.06)$ and decreased breakdown $(-11 \% ; p<0.08)$. The WOMAC index increased from 45 to 77 points, and VAS pain decreased from 73 to $31 \mathrm{~mm}$ (both $p<0.001$ ).

Conclusions Joint distraction can induce tissue structure modification in knee $\mathrm{OA}$ and could result in clinical benefit. No current treatment is able to induce such changes. Larger, longer and randomised studies on joint distraction are warranted.

Osteoarthritis $(\mathrm{OA})$ is a degenerative joint disorder characterised by progressive cartilage damage and loss, changes in bone and other periarticular tissues and commonly also, secondary joint inflammation. These changes in tissue structure are associated with pain, stiffness and functional disabilities. ${ }^{1}$

Knee OA affects roughly $6 \%$ of the adult population and is the most common form of OA, with a huge socioeconomic and healthcare burden. ${ }^{2}$

Few options are available for treatment of endstage knee $\mathrm{OA}$ and none have clearly been shown to halt or even reverse tissue structure damage. ${ }^{3}$ Removal of pain by replacing the destroyed joint with an endoprosthesis is the currently accepted treatment option for severe knee OA. Consequently, the number of total knee prostheses is exponentially increasing in the Western world and causes major economic burden. ${ }^{4} 5$ Over $40 \%$ of all knee replacements and up to $44 \%$ of all total knee revisions are performed in patients aged under $65 .^{6}$ Importantly, the procedure has a higher risk of failure in younger patients than in older patients. ${ }^{7} 8$ As such, development of alternative treatment strategies for severe knee OA, specifically those that can postpone a first prosthesis, are urgently needed.

Joint distraction is a surgical procedure in which the two bony ends of a joint are gradually separated to a certain extend for a certain period of time. Initially, joint distraction was used in the treatment of joint malalignment and joint contracture. An external fixation frame was used to actively reposition the joint and to increase the range of motion. Distraction was performed to prevent damage (compression) of the joint cartilage during the forced repositioning. In some of these patients $\mathrm{OA}$ was present in the treated joint and an unexpected clinical improvement of the OA was observed. 910 These clinical observations led us to a proof-of-concept study examining the benefit of joint distraction, by treating young patients with severe ankle OA. ${ }^{11}$ Two-thirds of patients treated for 3 months with joint distraction experienced significant clinical benefits for a period of up to 10 years. ${ }^{12}$ Based on preliminary radiographic outcome in a limited number of patients, it was suggested that joint distraction may lead to tissue structure modification as well.

We aimed to explore whether joint distraction can halt or reverse joint degeneration in knee OA in those cases where joint replacement surgery is indicated, and whether it has the potential to delay knee replacement surgery in relatively young patients in an open, uncontrolled clinical trial.

\section{PATIENTS AND METHODS \\ Patients}

Twenty patients with knee OA and with an indication for knee replacement surgery were included between 2006 and 2008 according to the following criteria: age $<60$ years, Visual Analogue Scale (VAS) of pain $\geq 60 \mathrm{~mm}$, radiographic signs of joint damage and primarily tibiofemoral $\mathrm{OA}$ (not patella-femoral OA). Exclusion criteria were severe symptoms in both knees, a history of inflammatory or septic arthritis and severe knee malalignment requiring surgical correction $\left(>10^{\circ}\right)$. Patients had been referred from peripheral hospitals to our academic hospital for a second opinion because 
indicated joint replacement surgery was refused by the patient or the patient's age prevented orthopaedic surgeons from carrying out knee prosthetic surgery. The study was approved by the medical ethical review committee of the University Medical Center Utrecht (No 04/086). All patients gave written informed consent.

\section{Distraction method}

Two monotubes with internal coil springs (Stryker, Monotube Triax) were placed parallel on the medial and lateral side, bridging the knee joint (figure 1A). Each monotube was fixed to two bone pins (Stryker, $6 \mathrm{~mm}$ self-drilling half pins) on each end and they were lengthened $2 \mathrm{~mm}$, all under anaesthesia. Pinholes were placed as far as possible from the joint line in order not to compromise the area needed for possible future prosthesis surgery. Over the following 3 days the joint was distracted twice a day by $0.5 \mathrm{~mm}$, bringing the total distraction to $5 \mathrm{~mm}$, which was confirmed by x-ray examination, and adjusted if necessary. After instructions about pin-site care, daily exercise and physical therapy, the patients were discharged from hospital. Patients were encouraged to load the distracted joint, with full weight bearing allowed.

Every 2 weeks the patients returned to the hospital. and the monotubes were removed temporarily. For 3-4 h, the knee was bent in a continuous passive motion device, pain at the pin sites determining the maximum degree of flexion; on average, $25^{\circ}$ $\left(15-80^{\circ}\right)$ flexion and full extension was reached. The monotubes were replaced and sufficient distraction was confirmed by x-ray examination and adjusted if needed.

After 2 months, the tubes and pins were removed at day care and patients went home without imposed functional restrictions. A continuous passive motion device was provided at their home to practice flexion of the knee joint. After reaching $90^{\circ}$ flexion (approximately 1-2 months after removal of the frame), the patients were advised to gain muscle strength by, for example, cycling.

\section{Structural outcome}

Patients visited the outpatient clinic twice before treatment (baseline), every 2 weeks during treatment, and 3, 6 and 12 months after the start of the treatment.

\section{Radiographic analysis}

At all visits, weightbearing, semiflexed, posterior-anterior radiographic views were acquired for evaluation by knee images digital analysis (KIDA) software. ${ }^{13}$ No physical limitations in obtaining adequate semiflexed views were observed. KIDA analyses provided minimal and mean joint space width (JSW) in both compartments. Mean subchondral bone density was determined by measuring density in a total of 16 regions adjoined to the bone cartilage interface in both compartments in tibia and femur, normalising the grey scale to that of an aluminium stepwedge reference. Analyses were performed blinded to the order of acquisition and characteristics of the patients.

\section{Quantitative MRI analysis}

At baseline and at 12 months, MRI acquisition (1.5T Philips Achieva) was performed using sequences validated for quantitative measurement of cartilage morphology. ${ }^{14} 15$ Coronal images were used to segment the femorotibial cartilage plates and bone surface, the operator and quality control reader being blinded to the order of sequence (baseline vs follow-up). Cartilage parameters were computed using custom software (Chondrometrics, Ainring, Germany). The primary structural outcomes ${ }^{16}$ were thickness of cartilage over total area of bone (ThCtAB) and the percentage area of denuded bone (dABp). Secondary structural outcome parameter was thickness of cartilage over area of bone covered with cartilage (ThCcAB).

\section{Biomarker analysis}

Serum and urine samples were collected and stored at $-80^{\circ} \mathrm{C}$. Cartilage collagen type II synthesis and breakdown were
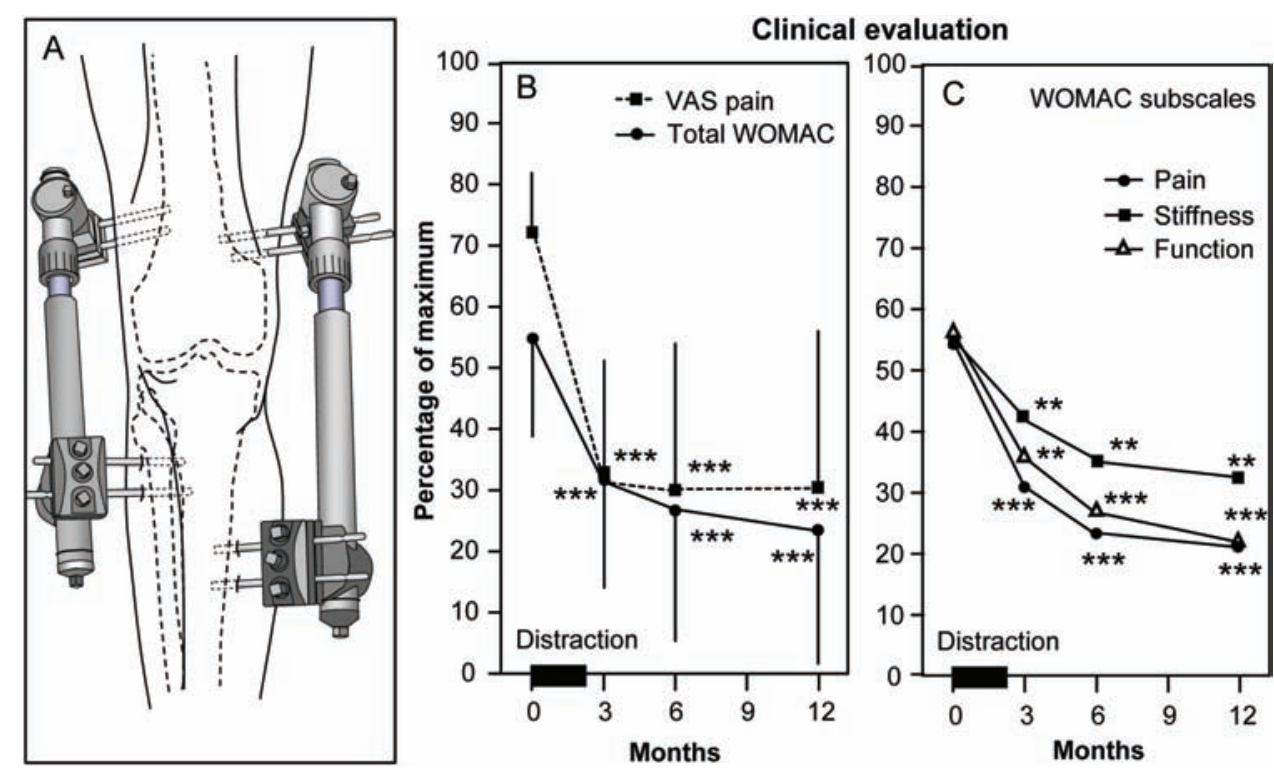

Figure 1 (A) Drawing of the monotubes placed on bone pins bridging the knee joint. Lengthening of the tubes (approximately $5 \mathrm{~mm}$ ) induces joint distraction. Springs within the tubes (like shock absorbers) allow restricted ( $3 \mathrm{~mm}$ ) axial movement without direct joint surface contact.

(B) Clinical evaluation presented by the total WOMAC and Visual Analogue Scale (VAS) pain, means \pm SD are given. Distraction is performed during the first 2 months, at 3 months (1 month after removal of the fixator) pain has decreased and the WOMAC score has increased, sustaining for at least 12 months. (C) WOMAC subscales. ${ }^{* *}$ Indicates statistical significance of $p<0.01,{ }^{* *} p<0.001$. WOMAC, Western Ontario and McMaster Universities Osteoarthritis Index. 
determined by serum N-propeptide of type IIA procollagen (PIIANP; Linco, EZPIIANP-53K) and urinary C-telopeptide of type II collagen (CTXII), Cartilaps; corrected for urine creatinine), respectively. Samples were analysed in duplicate, and longitudinal samples of one patient were assayed in one plate, to eliminate interkit variability.

\section{Clinical outcome}

The primary clinical outcome parameterwas the Western Ontario and McMaster Universities Osteoarthritis Index (WOMAC), ${ }^{17}$ normalised to a $100 \%$ scale, $100 \%$ being the worst condition. The secondary clinical outcome parameters were the VAS for pain (0-100 mm) and physical examination of the joint (pain on palpation, crepitus, pain with flexion and joint effusion).

\section{Statistical analysis}

Parametric statistics (two-sided paired t test) were used for all parameters to compare whether the follow-up values significantly differed from the baseline values. Spearman correlation coefficients were used to relate longitudinal changes at 1 year between parameters. Means \pm SDs are given and $p<0.05$ was considered a statistically significant difference.

\section{RESULTS}

Twenty-three patients were considered for treatment; one was excluded because of bilateral $\bigcirc A$, one was excluded because of remaining metal in the knee after anterior cruciate ligament reconstruction, and one withdrew from treatment after inclusion. Of the 20 patients included, aged $48 \pm 7$ years, 11 were men. Eleven left knees and nine right knees were treated. Eighteen patients had predominantly $\mathrm{OA}$ in the medial compartment while two patients had OA in the lateral compartment. Three, four, 11 and two patients had a baseline Kellgren and Lawrence $(\mathrm{K} \& \mathrm{~L}) \operatorname{grade}^{18}$ of $1,2,3$ and 4 , respectively. The average body mass index was 30 (range 25-36). Of the 20 patients 16 had had previous knee surgery. In one case anterior cruciate ligament reconstruction was carried out, in four patients a tibial osteotomy was performed and in 15 patients an arthroscopy was performed, 12 of those had a partial meniscectomy or meniscopexy. All surgery took place more than 1 year before distraction and without satisfactory results.

\section{Complications}

Two patients had lung emboli despite appropriate anticoagulative prevention (nadroparin). Patients were admitted to hospital for a week and given anticoagulative treatment (nadroparin), after which they were discharged in good condition continuing treatment (acenocoumarol) for 6 months. Of the 20 patients, 17 had single or multiple pin tract infections. All were successfully treated with antibiotics (flucloxacillin) for an average of 4 weeks. One patient had to be admitted to the hospital for 1 week to receive antibiotics intravenously. None of the patients had any signs of osteomyelitis.

\section{Structural outcome}

Radiographic analysis

The mean JSW of the most affected compartment increased from $2.7 \pm 1.7$ to $3.6 \pm 1.2 \mathrm{~mm}$ from baseline to 12 months $(\mathrm{p}<0.05$; figure 2 top left). The minimum JSW increased from $1.0 \pm 1.2$ to $1.9 \pm 1.3 \mathrm{~mm}(\mathrm{p}<0.01)$. Subchondral density at baseline
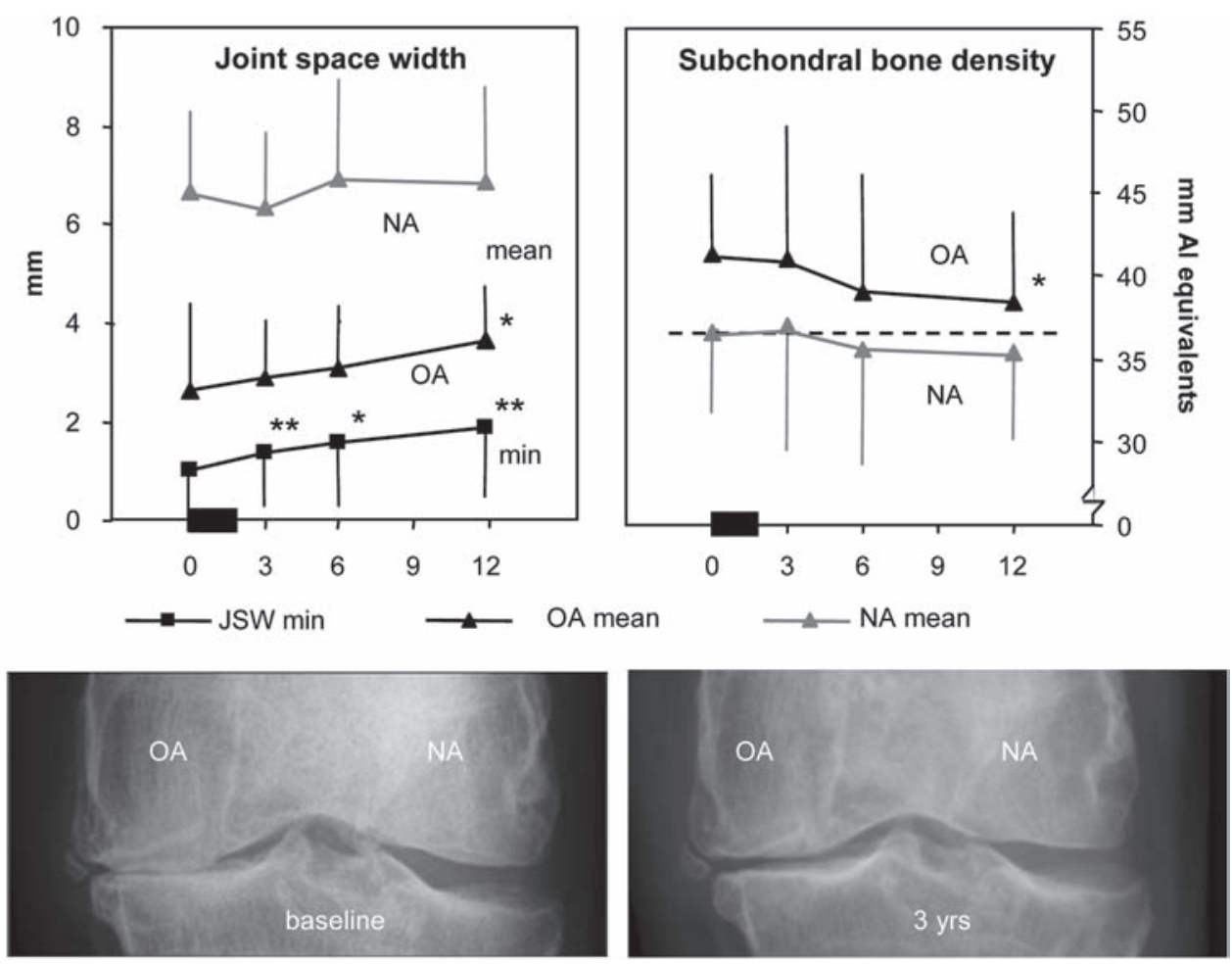

Figure 2 Joint space width (JSW) (KIDA measurement; mean \pm SD). Minimum JSW (min) continuously increased after distraction. The mean JSW of the affected (OA) compartment also increased over time. The mean JSW of the less affected compartment (NA), did not change over time. Subchondral bone density (KIDA, as mm aluminium (Al) equivalents; using a reference). The affected (OA) compartment showed a decrease in bone density, the less affected compartment (NA) did not. ${ }^{*} p<0.05,{ }^{* *} p<0.01$. Representative radiographs before and 3 years after distraction; clear increase in JSW in affected (OA) compartment. KIDA, Knee Images Digital Analysis; OA, osteoarthritis. 
was higher in the most affected compartment than it was in the contralateral compartment: $41 \pm 5$ and $37 \pm 1 \mathrm{~mm}$ aluminium equivalents, respectively (figure 2 top right), and had decreased $5.8 \pm 12 \mathrm{~mm}$ aluminium equivalents $(\mathrm{p}<0.05)$ in the affected compartment and $2.6 \pm 5 \mathrm{~mm}$ in the less affected compartment at 1 year of follow-up.

\section{Quantitative MRI analysis}

Quantitative MRI analysis at 1 year showed an increase in mean ThCt $\mathrm{AB}$ of the most affected compartment from $2.4 \pm 0.6$ to $3.0 \pm 0.5 \mathrm{~mm}(\mathrm{p}<0.001)$ and a decrease of mean dABp from $22 \pm 20 \%$ to $5 \pm 9 \%(p<0.001)$ (figure 3 ; including a representative pretreatment and post-treatment MRI image). ThCcAB showed a borderline increase from $2.9 \pm 0.3$ to $3.1 \pm 0.4 \mathrm{~mm}(p=0.062)$,

meaning that despite the increase in cartilage area, the overall average thickness did not decrease, which only occurs when either the newly formed cartilage is just as thick as surrounding cartilage or surrounding cartilage also thickens. Results for separate compartments (femur and tibia; and the less affected compartment) and whole joint are provided in table 1.

\section{Biomarkers}

Biomarkers showed an initial high increase during distraction, normalising 1 month after distraction (data not shown). Changes between 6 and 12 months' follow-up showed a trend towards a decrease of collagen type II breakdown marker CTXII $(-11 \pm 39 \% ; p=0.078)$ and an increase of collagen type II synthesis marker PIIANP $(+103 \pm 298 \% ; p=0.060)$. The mean change in
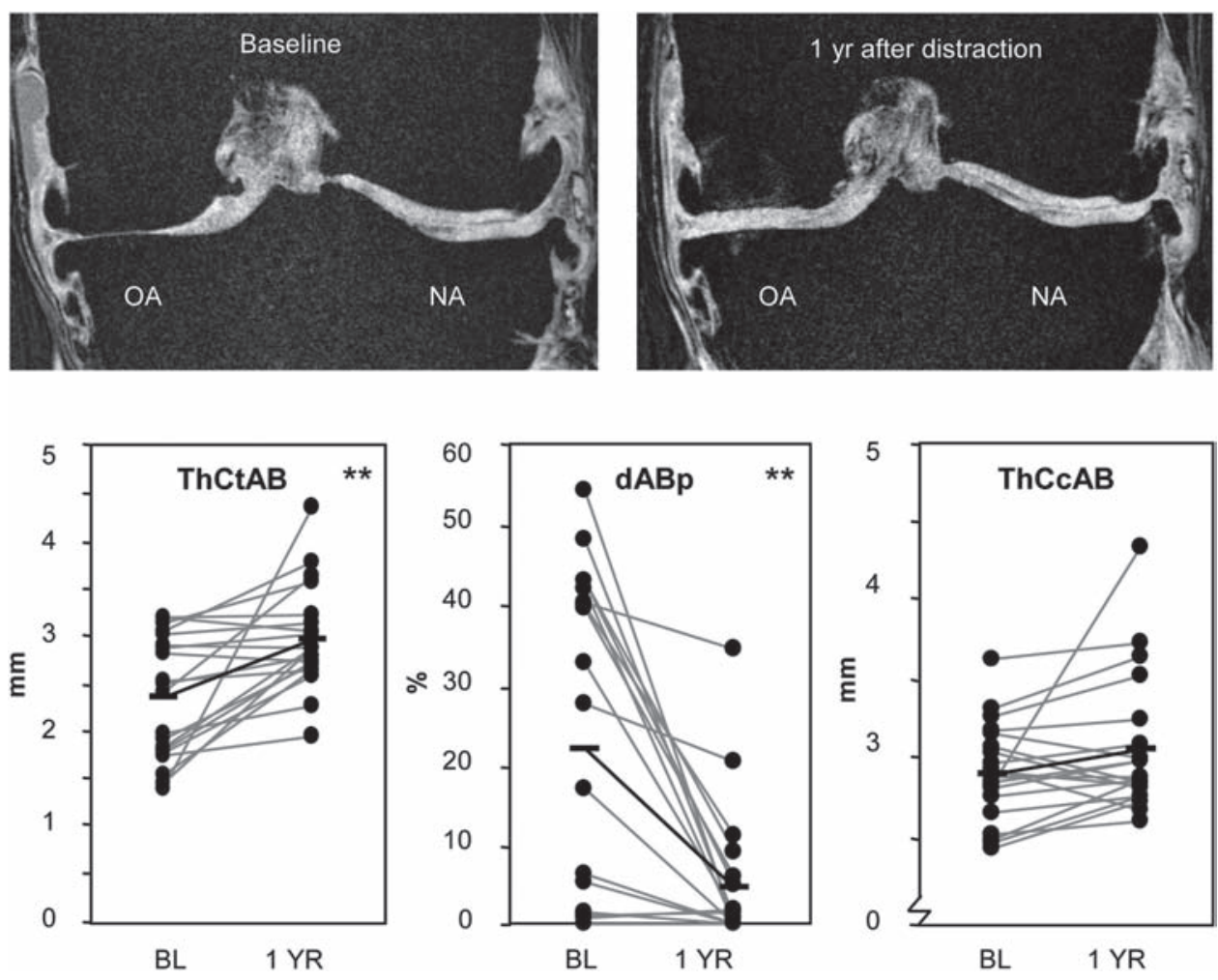

Figure 3 Representative image of single slides before and 1 year after treatment, showing an increase in cartilage tissue in the affected compartment. Quantitative MRI analysis of cartilage of the affected compartment of the individual 20 patients (grey lines) at baseline (BL) and after 1 year of follow-up (1 YR). Black lines indicate mean values. ThCtAB, thickness of cartilage over total area of bone; dABp, area of denuded bone (both $\left.{ }^{* *} p<0.01\right)$; ThCcAB, thickness of cartilage over area of bone covered with cartilage $(p<0.062)$.

Table 1 MRI outcome for femoral and tibial side of the most affected compartment (OA, osteoarthritis) and the less affected compartment (NA, not affected) as well as for the whole joint (both compartments) of 20 patients treated for 2 months with joint distraction, before distraction (BL, baseline) and after 1 year, including two-sided $p$ values

\begin{tabular}{|c|c|c|c|c|c|c|c|c|c|}
\hline \multirow[b]{2}{*}{ MRI } & \multicolumn{3}{|l|}{$O A$} & \multicolumn{3}{|l|}{ NA } & \multicolumn{3}{|l|}{ Whole joint } \\
\hline & $\overline{\mathrm{BL}}$ & 1 Year & $\bar{p}$ Value & BL & 1 Year & $p$ Value & $\overline{B L}$ & 1 Year & p Value \\
\hline \multicolumn{10}{|c|}{ ThCtAB (mm) } \\
\hline Femur & $1.00 \pm 0.41$ & $1.41 \pm 0.30$ & 0.000 & $2.10 \pm 0.42$ & $2.11 \pm 0.41$ & 0.598 & $3.25 \pm 0.39$ & $3.57 \pm 0.47$ & 0.001 \\
\hline \multicolumn{10}{|l|}{$\mathrm{dABp}(\%)$} \\
\hline Femur & $27.31 \pm 25.64$ & $4.19 \pm 10.22$ & 0.000 & $0.63 \pm 1.40$ & $0.50 \pm 1.39$ & 0.402 & $11.32 \pm 10.02$ & $2.52 \pm 4.44$ & 0.001 \\
\hline Tibia & $16.70 \pm 17.22$ & $4.82 \pm 8.33$ & 0.006 & $0.65 \pm 2.34$ & $0.57 \pm 2.54$ & 0.592 & & & \\
\hline Tibia & $1.62 \pm 0.24$ & $1.64 \pm 0.25$ & 0.653 & $2.07 \pm 0.30$ & $2.07 \pm 0.25$ & 0.698 & & & \\
\hline
\end{tabular}

Primary structural outcome parameters: thickness of cartilage over total area of bone (ThCtAB) and the percentage area of denuded bone (dABp); secondary structural outcome parameter: thickness of cartilage over area of bone covered with cartilage (ThCcAB). Mean \pm SD values are presented. 
the ratio of PIIANP/CTXII between 6 and 12 months suggested a net increase in collagen synthesis $(p=0.056)$.

\section{Clinical outcome}

The total WOMAC index questionnaire decreased from $55 \pm 16$ points at baseline to $23 \pm 21$ points at 1 year ( $<<0.001$; figure $1 B$ ). Of the 20 patients, 18 showed an improvement of $>10 \%$ and 16 of $>25 \%$. The individual components of the WOMAC index (pain, stiffness and function) all improved significantly $(p<0.001$; figure 1C). VAS pain decreased from $73 \pm 9 \mathrm{~mm}$ at baseline to $31 \pm 26 \mathrm{~mm}(\mathrm{p}<0.001)$ at 1 year (figure $1 \mathrm{~B})$. Physical examination of the knee showed an improvement from $46 \pm 22 \%$ to $75 \pm 24 \%$ $(p<0.001)$ of the maximum score (data not shown).

\section{Correlation between structural parameters}

All MRI parameters correlated positively and significantly with the increase in mean radiographic JSW (all $r>0.51$ and $p<0.01$ ). The increase in collagen type II synthesis marker PIIANP between 6 and 12 months correlated with the change in ThCtAB and $\mathrm{dABp}$ (figure 4). CTXII change did not show such correlations. There were no clear correlations between structural and clinical parameters.

Individual results are shown in the online supplementary data.

\section{DISCUSSION}

This exploratory prospective open uncontrolled study demonstrates, for the first time, that joint distraction can reverse tissue structure damage in patients with knee OA considered for joint replacement surgery, in addition to providing significant clinical benefit. Over a period of 1 year, 2 months of knee distraction significantly increased radiographic JSW on weightbearing radiographs, to increase cartilage thickness and decrease denuded bone area as evaluated by MRI, and to increase the ratio of cartilage collagen type II synthesis over breakdown as determined by biomarker analyses. Next to these significant tissue structural changes, improvement of functional abilities and reduction of pain was observed. No other treatment at present can induce such changes at this stage of the disease, and no treatment is currently approved for structural modification in OA at any stage.

Although the clinical effects are significant, the possibility of a placebo effect cannot be ruled out. On the other hand, placebo effects are known to be highest during and directly after treatment and to significantly decrease when treatment stops. In this study a progressive pattern of clinical benefits up to 1 year was seen, which is highly unlikely to represent a placebo effect. Unfortunately, registration of medication (before and after treatment) was not adequately performed. Although medication use is unlikely to explain the structural changes the possibility of some influence on the clinical outcome cannot be ruled out. This is a flaw in the study set-up.

Regenerative medicine focuses on creating circumstances under which damaged tissue recovers. This study is the first to demonstrate intrinsic tissue structure repair in OA. Historically, the regenerative capacity of cartilage has been questioned owing to the slow turnover rate of cartilage matrix, especially of collagen. ${ }^{19}$ However, this study shows that a significant amount of cartilage tissue is formed within 1 year after the distraction, demonstrating that under certain conditions cartilage has regenerative capacity.

It should be noticed that the rate of cartilage thickening $(+0.9 \mathrm{~mm} /$ year $)$ in this study is surprisingly fast. In general, it has been observed that the breakdown of cartilage does not exceed an average rate of $0.2 \mathrm{~mm} /$ year. $^{20}$ A technical artefact is not plausible since a similar change in the non-affected compartment would have been expected. Moreover, there is a gradual increase over time after distraction, again not in favour of an artefact. Although no histological or biochemical analysis of tissue quality could be performed in this study, indirect evidence suggests that the cartilage is of sustainable quality. X-ray examinations were carried out under full weightbearing conditions, demonstrating the mechanical competence of the formed tissue. In the first patients treated, $\mathrm{x}$-ray evaluation several years after distraction demonstrates a sustained gain in JSW over time. Also, the increase in the ratio of collagen type II synthesis/breakdown and the positive correlation between collagen type II synthesis and increase in the cartilage thickness on MRI, support formation of functionally relevant hyaline (type II collagen-containing) cartilage. Nevertheless, future animal and clinical studies (including qualitative MRI parameters based on, for example, delayed gadolinium-enhanced MRI of cartilage ${ }^{21}$ will have to explore the compositional properties of the newly formed tissue.

In addition, the question arises, what might the underlying mechanism of the observed structure repair be? It is hypothesised that the temporary distraction prevents mechanical stress on the cartilage, prevents further wear and tear and allows tissue repair to begin. Joint fluid pressure changes are

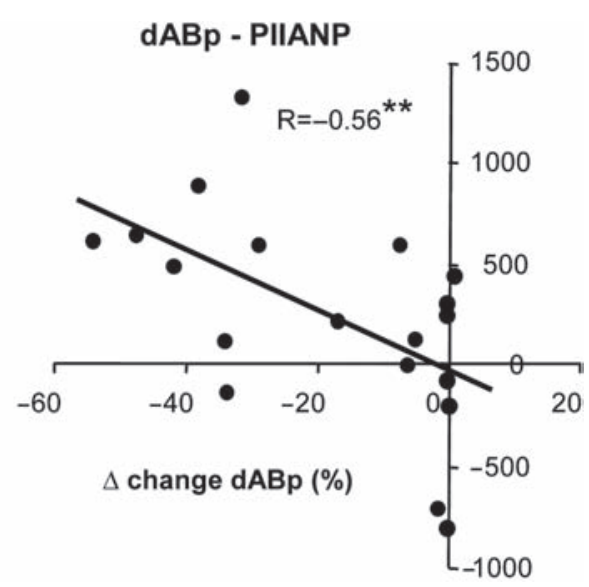

Figure 4 Correlations between the changes (compared with baseline) in cartilage thickness (ThCtAB; mm) and area of denuded bone (dABp; \%) on $\mathrm{MRI}$ and the change in serum N-propeptide of type IIA procollagen (PIIANP; ng/ml) between 6 months and 1 year of follow-up. ${ }^{* *} \mathrm{p}<0.01$. ThCtAB, thickness of cartilage over total area of bone. 
maintained during the distraction period, because the springs in the distraction tubes allow limited axial oscillation during loading and unloading of the distracted joint. These fluid pressure oscillations may provide nutrition and may trigger the cartilage cells to initiate tissue repair (re-differentiation of the diseased chondrocytes). ${ }^{22} 23$ During distraction, the load on the bone (the biomechanical trigger for normal bone formation) is transferred through the frame instead of the subchondral bone, leading to subchondral bone resorption, which subsequently normalises after distraction. This significant bone turnover may trigger the release of growth factors as bone matrix provides a store of resident growth factors such as transforming growth factors $\beta$, bone morphogenetic proteins and insulin-like growth factors that stimulate cartilage tissue repair. ${ }^{24} 25$

Treatment in this study was accompanied by two major safety concerns. In two out of 20 patients, a pulmonary embolism developed. In retrospect, in both patients there was a family history of venous thrombosis. In future studies additional attention should be paid to this severe complication in anamnesis. A higher dose of anticoagulative agents could be considered. In 17 out of 20 patients pin tract infections developed, which could be treated adequately with antibiotics. Pin tract infections are a general complication of the application of external fixators and did not result in deep infections. Although these patients might have prosthetic surgery in the future, the risk of infection was minimised by placing bone pins outside the expected future operating area. Nonetheless, more attention should be paid to reducing the number of these complications in future studies.

It is currently unclear which group of patients would benefit best from this treatment. In this study, only young patients (<60 years) with severe OA considered for joint replacement surgery were treated. Selection by referral from peripheral hospitals might have resulted in an inclusion bias. Thus, results from this population cannot be generalised to all patients considered for prosthetic surgery. This patient group showed a diversity of OA stages with K\&L grade varying between 1 and 4 . In general practice patients with a low K\&L grade but significant joint pain are also considered for knee replacement surgery; this has been the subject of a recent discussion. ${ }^{26}$ Overall, patients showed a positive change in structural parameters but effects were variable. Unfortunately, group size does not allow valid analyses to identify predictive factors for clinical or structural benefit. Potential relations found may depend on coincidence. Prediction of efficacy needs to be examined in future larger studies.

The primary goal of this intervention is to postpone joint replacement in relatively young patients, in order to reduce the potential numbers of revision operations. Larger (and longer) trials in a variety of $\mathrm{OA}$ populations need to be performed to optimise the distraction treatment (duration), and to identify those patients who would profit the most, and for the longest period of time, from the treatment.

At present, distraction is the only treatment that can reverse cartilage tissue structure damage in severe knee $\mathrm{OA}$ and at the same time results in significant clinical improvement.

Acknowledgements Marja Lafeber is acknowledged for analysing the x-ray data with use of KIDA.

Funding This study was financially supported by the Dutch Arthritis Foundation. Competing interests None.
Ethics approval This study was conducted with the approval of the University Medical Center Utrecht.

Provenance and peer review Not commissioned; externally peer reviewed.

\section{REFERENCES}

1. Moskowitz RW. Osteoarthritis. 4th edition. Philadelphia, PA: Lippincott Williams \& Wilkins 2007.

2. Felson DT, Lawrence RC, Dieppe PA, et al. Osteoarthritis: new insights. Part 1: the disease and its risk factors. Ann Intern Med 2000;133:635-46.

3. Lohmander LS, Roos EM. Clinical update: treating osteoarthritis. Lancet 2007;370:2082-4.

4. Bitton R. The economic burden of osteoarthritis. Am J Manag Care 2009;15(8 Suppl):S230-5.

5. Losina E, Walensky RP, Kessler CL, et al. Cost-effectiveness of total knee arthroplasty in the United States: patient risk and hospital volume. Arch Intern Med 2009;169:1113-21; discussion 21-2.

6. Kurtz SM, Lau E, Ong K, et al. Future young patient demand for primary and revision joint replacement: national projections from 2010 to 2030. Clin Orthop Relat Res 2009;467:2606-12.

7. W-Dahl A, Robertsson 0 , Lidgren L. Surgery for knee osteoarthritis in younger patients. Acta Orthop 2010;81:161-4.

8. Santaguida PL, Hawker GA, Hudak PL, et al. Patient characteristics affecting the prognosis of total hip and knee joint arthroplasty: a systematic review. Can J Surg 2008;51:428-36.

9. van Roermund PM, van Valburg AA, Duivemann E, et al. Function of stiff joints may be restored by llizarov joint distraction. Clin Orthop Relat Res 1998;348:220-7.

10. van Valburg AA, van Roermund PM, Lammens J, et al. Can llizarov joint distraction delay the need for an arthrodesis of the ankle? A preliminary report. J Bone Joint Surg Br 1995; 77:720-5.

11. Marijnissen AC, Van Roermund PM, Van Melkebeek J, et al. Clinical benefit of joint distraction in the treatment of severe osteoarthritis of the ankle: proof of concept in an open prospective study and in a randomized controlled study. Arthritis Rheum 2002;46:2893-902.

12. Ploegmakers JJ, van Roermund PM, van Melkebeek J, et al. Prolonged clinical benefit from joint distraction in the treatment of ankle osteoarthritis. Osteoarthr Cartil 2005; 13:582-8.

13. Marijnissen AC, Vincken KL, Vos PA, et al. Knee Images Digital Analysis (KIDA): a novel method to quantify individual radiographic features of knee osteoarthritis in detail. Osteoarthr Cartil 2008;16:234-43.

14. Burgkart R, Glaser C, Hyhlik-Dürr A, et al. Magnetic resonance imaging-based assessment of cartilage loss in severe osteoarthritis: accuracy, precision, and diagnostic value. Arthritis Rheum 2001;44:2072-7.

15. Eckstein F, Charles HC, Buck RJ, et al. Accuracy and precision of quantitative assessment of cartilage morphology by magnetic resonance imaging at 3.0T. Arthritis Rheum 2005:52:3132-6.

16. Buck RJ, Wyman BT, Le Graverand MP, et al.; A9001140 Investigators. An efficient subset of morphological measures for articular cartilage in the healthy and diseased human knee. Magn Reson Med 2010;63:680-90.

17. Bellamy N. Outcome measurement in osteoarthritis clinical trials. J Rheumatol Supp/ 1995:43:49-51.

18. Kellgren JH, Lawrence JS. Radiological assessment of osteo-arthrosis. Ann Rheum Dis 1957;16:494-502.

19. Hunziker EB. Articular cartilage repair: basic science and clinical progress. A review of the current status and prospects. Osteoarthr Cartil 2002;10:432-63.

20. Emrani PS, Katz JN, Kessler CL, et al. Joint space narrowing and Kellgren-Lawrence progression in knee osteoarthritis: an analytic literature synthesis. Osteoarthr Cartil 2008;16:873-82.

21. Burstein D, Gray M, Mosher T, et al. Measures of molecular composition and structure in osteoarthritis. Radiol Clin North Am 2009;47:675-86.

22. Lafeber F, Veldhuijzen JP, Vanroy JL, et al. Intermittent hydrostatic compressive force stimulates exclusively the proteoglycan synthesis of osteoarthritic human cartilage. Br J Rheumatol 1992;31:437-42.

23. van Valburg AA, van Roy HL, Lafeber FP, et al. Beneficial effects of intermittent fluid pressure of low physiological magnitude on cartilage and inflammation in osteoarthritis. An in vitro study. J Rheumatol 1998;25:515-20.

24. van der Kraan PM, Buma P, van Kuppevelt T, et al. Interaction of chondrocytes, extracellular matrix and growth factors: relevance for articular cartilage tissue engineering. Osteoarthr Cartil 2002;10:631-7.

25. Westacott CI, Webb GR, Warnock MG, et al. Alteration of cartilage metabolism by cells from osteoarthritic bone. Arthritis Rheum 1997;40:1282-91.

26. Conaghan PG, D’Agostino MA, Le Bars M, et al. Clinical and ultrasonographic predictors of joint replacement for knee osteoarthritis: results from a large, 3-year, prospective EULAR study. Ann Rheum Dis 2010;69:644-7. 